Discussion

\title{
Exchange of comments on "Temporal shifting: a hidden key to the skewed peak puzzle"
}

\author{
Su-Cheng Pai ${ }^{\text {a,* }}$, Ling-Yun Chiao ${ }^{\text {b }}$
}

a Division of Marine Chemistry, Institute of Oceanography, National Taiwan University, Taipei, Taiwan

${ }^{\mathrm{b}}$ Division of Marine Geology and Geophysics, Institute of Oceanography, National Taiwan University, Taipei, Taiwan

Available online 19 March 2007

Keywords: Flow injection analysis; Linear chromatography; Peak shape; Temporal shift

Soon after our paper [1] was published, a response arrived from Felinger [2] who strongly suggested that one should use the first moment to locate the correct peak position. The argument then, becomes "how can one find the correct spatial first moment from a skewed peak on a time-based recorder chart?"

In our work, we have assumed that the sample zone is spatially Gaussian and temporally carrying a tail; and we have defined a parameter $\Phi$ (in s units) which represents the shift of an apparent peak apex $\left(t_{\mathrm{p}}^{*}\right)$ from its true elution time $\left(t_{\mathrm{p}}\right.$ as calculated by $t_{\mathrm{p}}=L / u$, where $L$ is the length of tubular channel and $u$ the flow speed):

$\Phi=t_{\mathrm{p}}-t_{\mathrm{p}}^{*}$

As $t_{\mathrm{p}}$ increases, the solution for $\Phi$ gradually converges to a fixed threshold. Thus, one may use the following approximation to evaluate the scale of the shift:

$\Phi \approx \frac{D}{u^{2}}$

Thus, the temporal peak apex should be at

$t_{\mathrm{p}}^{*} \approx \frac{L}{u}-\Phi$

Apart from this, other parameters can also be obtained. For example, one may calculate the time for the mean peak area (denoted as $t_{\mathrm{a}}^{*}$ ) and the temporal first moment (denoted as $t_{\mathrm{r}}^{*}$ ). For the former, we let

$\left.\int c(t) \mathrm{d} t\right|_{t=t_{\mathrm{a}}^{*}}=\frac{A_{t}}{2}$

\footnotetext{
* Corresponding author. Tel.: +886 2 23627358; fax: +8862 23632912

E-mail address: scpai@ntu.edu.tw (S.-C. Pai).
}

where $A_{t}$ is the peak area measured on the $t$ axis (in conc-s units). The solution for $t_{\mathrm{a}}^{*}$ can be approximated as

$t_{\mathrm{a}}^{*}=\frac{L}{u}+\Phi$

For the latter, we use the following equation:

$t_{\mathrm{r}}^{*}=\frac{1}{A_{t}} \int t c(t) \mathrm{d} t$

and the solution is

$t_{\mathrm{r}}^{*}=\frac{L}{u}+2 \Phi$

Thus, $t_{\mathrm{p}}^{*}$ and $t_{\mathrm{a}}^{*}$ lie aside the spatial first moment $t_{\mathrm{p}}$ with an almost equal distance $(\Phi)$; whereas $t_{\mathrm{r}}^{*}$ is located at an even later time at $t_{\mathrm{p}}+2 \Phi$. These solutions have been verified numerically and demonstrated in Fig. 1(A-C).

Neither $t_{\mathrm{p}}^{*}, t_{\mathrm{a}}^{*}$ nor $t_{\mathrm{r}}^{*}$ represents the correct elution time (or the spatial first moment), but we can use either one to construct a temporal peak after finding an empirical $\Phi$ and compensating for the corresponding position shifts in a Gaussian equation. Thus, all three equations shown below can be used to restore an experimental peak.

$$
\begin{aligned}
& c(t)=\frac{A_{t}}{\sqrt{4 \pi \Phi t}} \mathrm{e}^{-\left(t-t_{\mathrm{p}}^{*}-\Phi\right)^{2} /(4 \Phi t)} \\
& c(t)=\frac{A_{t}}{\sqrt{4 \pi \Phi t}} \mathrm{e}^{-\left(t-t_{\mathrm{a}}^{*}+\Phi\right)^{2} /(4 \Phi t)} \\
& c(t)=\frac{A_{t}}{\sqrt{4 \pi \Phi t}} \mathrm{e}^{-\left(t-t_{\mathrm{r}}^{*}+2 \Phi\right)^{2} /(4 \Phi t)}
\end{aligned}
$$

There is no doubt that the first one (i.e. Eq. (8)) is always the easiest and most accurate (free from baseline noise) to serve this purpose. Examples have been given in another published paper [3]. 

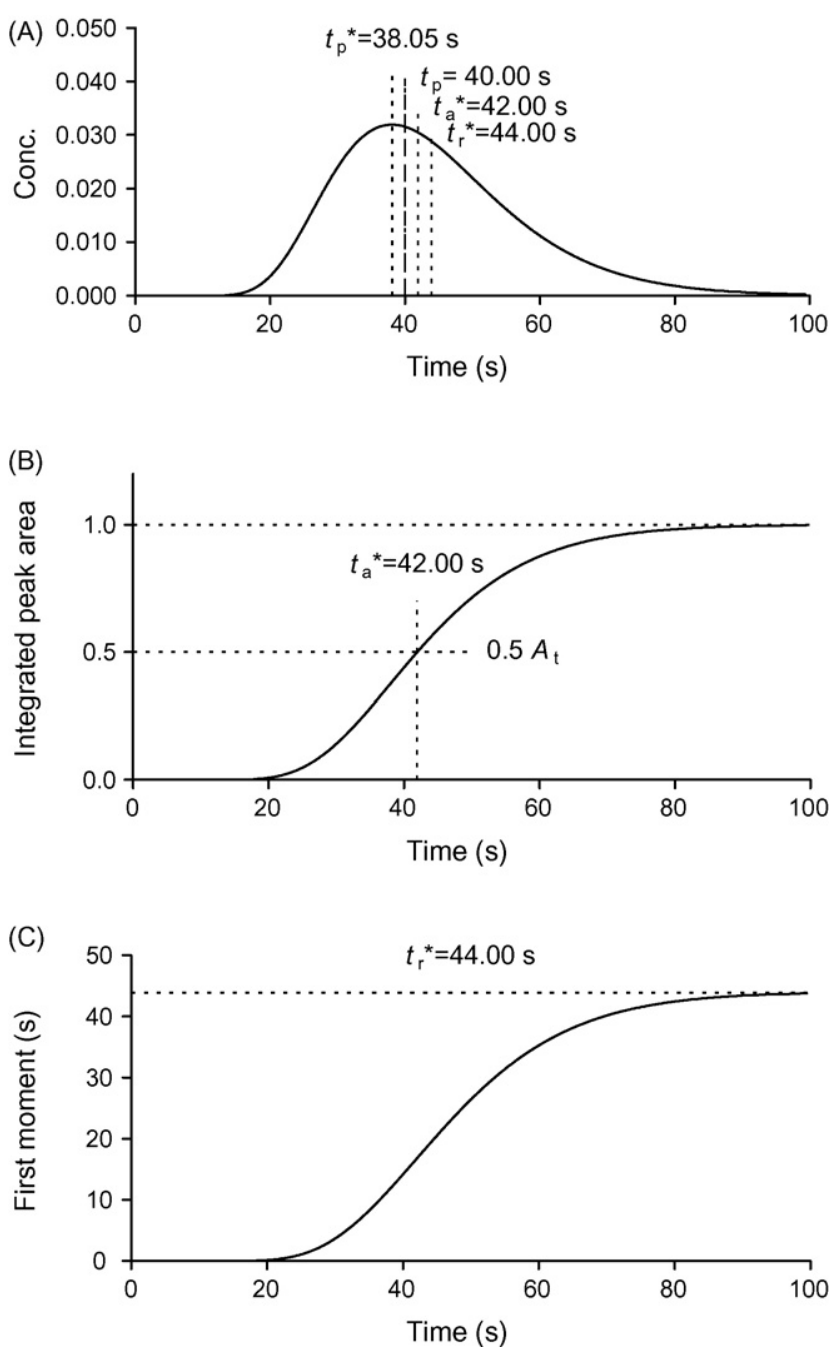

Fig. 1. (A) A skewed temporal curve is plotted from the data of Ref. [1] Fig. 3 in which the channel length is $L=40 \mathrm{~cm}$, the temporal peak area $A_{t}=1$ conc-s, the flow speed $u=1 \mathrm{~cm} \mathrm{~s}^{-1}$, the expansion coefficient $\mu_{\mathrm{t}}=2 \mathrm{~s}^{1 / 2}$ and the dispersion coefficient $D=2 \mathrm{~cm}^{2} \mathrm{~s}^{-1}$. The true retention time is $t_{\mathrm{p}}=L / u=40 \mathrm{~s}$, the apparent peak apex is found at $t_{\mathrm{p}}^{*}=38.05 \mathrm{~s}$, the peak area mean is at $t_{\mathrm{a}}^{*}=42.00 \mathrm{~s}$, whereas the first moment is at $t_{\mathrm{r}}^{*}=44.00 \mathrm{~s}$. The shifts from $t_{\mathrm{p}}$ to either $t_{\mathrm{p}}^{*}$ or $t_{\mathrm{a}}^{*}$ are almost identical in scale but opposite in signs and can be approximated by $\Phi=D / u^{2}$, the shift for $t_{\mathrm{r}}^{*}$ is $2 \Phi$. (B) The position of the peak area mean $\left(t_{\mathrm{a}}^{*}\right)$ is obtained by taking the cross point for the half integrated peak area. (C) Integrated calculation shows that the first moment $t_{\mathrm{r}}^{*}$ is $44.00 \mathrm{~s}$.

Felinger calculated the data provided from Fig. 3 of Ref. [1], but he used an erroneous diffusivity of $D=4 \mathrm{~cm}^{2} \mathrm{~s}^{-1}$, thus giving an elution time of $48 \mathrm{~s}$ as opposed to $40 \mathrm{~s}$ calculated from the spatial distribution. In that example, the temporal expansion coefficient was designated as $\mu_{\mathrm{t}}=2 \mathrm{~s}^{1 / 2}$, which is equivalent to a diffusivity of $D=2 \mathrm{~cm}^{2} \mathrm{~s}^{-1}$ (i.e. $D=0.5 \mu_{\mathrm{t}}^{2} u^{2}$ ). The results of our calculation (also see Fig. 1) show that the temporal peak apex is at $t_{\mathrm{p}}^{*} \approx 38 \mathrm{~s}$, a shift of $\approx-2 \mathrm{~s}$ from $t_{\mathrm{p}}$; the temporal peak area mean is at $t_{\mathrm{r}}^{*}=42 \mathrm{~s}$, a shift of $+2 \mathrm{~s}$ from $t_{\mathrm{p}}$. The temporal first moment is at $t_{\mathrm{r}}^{*}=44 \mathrm{~s}$, a shift of $+4 \mathrm{~s}$ from $t_{\mathrm{p}}$. There is no conceptual conflict between Felinger and us that "the temporal first moment gives a larger elution time than the spatial moment," or that "one should never use the location of the apex but the first moment."

Felinger introduced an alternative equation (Eq. (5) of Ref [2]) to describe the concentration profile based on temporal terms (e.g. using $\sigma_{\mathrm{t}}$ instead of $\sigma_{\mathrm{L}}$ ):

$c(z, t)=\frac{A}{\sqrt{4 D \pi t^{3}}} \mathrm{e}^{-(z-u t)^{2} / 4 D t}$

He claimed that the spatial first moment of this equation is $L / u$, and that the temporal first moment is the same. And, therefore, the shift does not exist. However, we think it does.

The first portion of Eq. (11) is supposed to be the peak height at $z=u t$. But, one should be aware that the physical dimension of $\sqrt{4 D \pi t^{3}}$ is "cm-s." If $A$ represents the peak area measured along the $t$ axis (i.e. $A_{t}$ in conc-s units), then, the physical dimension for the peak height would be "conc- $\mathrm{cm}^{-1}$, which is unrealistic.

In our derivation, the peak height at $\left(z=L, t=t_{\mathrm{p}}\right)$ should be

$h=c\left(L, t_{\mathrm{p}}\right)=\frac{A_{\mathrm{L}}}{\sqrt{4 D \pi t_{\mathrm{p}}}}$

Since $A_{t}=A_{\mathrm{L}} / u$ and $u=L / t_{\mathrm{p}}$, it can be expressed as

$h=c\left(L, t_{\mathrm{p}}\right)=\frac{A_{t}}{\sqrt{4 D \pi t_{\mathrm{p}}^{3} L^{-2}}}$

Therefore, the correct expression for Eq. (11) should be

$c(z, t)=\frac{A_{t}}{\sqrt{4 D \pi t^{3} z^{-2}}} e^{-(z-u t)^{2} / 4 D t}$

This equation is exactly the same as

$c(z, t)=\frac{A_{\mathrm{L}}}{\sqrt{4 D \pi t}} \mathrm{e}^{-(z-u t)^{2} / 4 D t}$

Therefore, the spatial first moments for both Eqs. (14) and (15) are $t_{\mathrm{p}}=L / u$, and the temporal first moments are all equal at $t_{\mathrm{r}}^{*}=L / u+2 \Phi$. The shift has not "disappeared."

Felinger also argued that the reasoning attributed to the temporal peak skewness is well understood and characterized. In fact, most users, except for those competent chromatographers, do normally regard the temporal peak apex as the true elution time. And, it is very difficult to explain to them and persuade them that these two times are different. Felinger's comments and this reply can help readers further clarify the temporal shifting problem.

\section{References}

[1] S.C. Pai, L.Y. Chiao, J. Chromatogr. A 1139 (2007) 104.

[2] A. Felinger, J. Chromatogr. A 1148 (2007) 260.

[3] S.C. Pai, Y.H. Lai, L.Y. Chiao, T. Yu, J. Chromatogr. A 1139 (2007) 109. 